Pacific Journal of Mathematics

AN ALGEBRAIC APPROACH TO EXTERIOR DIFFERENTIAL 


\title{
AN ALGEBRAIC APPROACH TO EXTERIOR DIFFERENTIAL SYSTEMS
}

\author{
H. H. JOHNSON
}

This paper concerns E. Cartan's theory of systems of exterior differential forms. We define a purely algebraic model which determines many of the system's properties. By algebraic constructions such concepts as "involutive", "characters" and "prolongations" are defined and the main theorems are given simple algebraic proofs. These methods are applied to characterize systems which reproduce themselves under prolongations. The prolongation theorem of Kuranishi is proved algebraically.

The purpose of this section is to explain the sources of the algebraic structures studied in the rest of the paper. E. Cartan initiated the study of exterior differential systems in [1, 2]. In [2] they were treated in a very algebraic manner which was used in Cartan's theory of infinite groups. Kuranishi in his prolongation theorem [6] again reduces problems to algebraic questions. We wish to isolate and exploit the purely algebraic characteristics of these systems even more fully in this paper.

Cartan studied systems of "Pfaffians", i.e., differential equations defined by a finite number of linearly independent real analytic 1-forms on an open ball in $E^{n}$. Let $A$ denote the module of real analytic 1forms over the ring $R$ of real analytic functions on a fixed open ball in $E^{n}$, Euclidean $n$-space. Consider $\wedge A=\wedge^{\circ} A+\wedge^{1} A+\cdots+\wedge^{n} A$, the exterior algebra generated by $A$ over $R$. Let $S$ and $I$ be submodules of $A . S$ is the given module of Pfaffians which define for Cartan partial differential equations. $I$ is called the module of independent variables. Let $(S)$ denote the ideal in $\wedge A$ generated by $S$. We use this ideal because if $S$ is zero on some integral submanifold of $E^{n}$, so is every element of $(S)$. Let $d$ denote the exterior derivative.

Choose a linear subspace $T$ of $A$ so that $A$ is the direct sum of $I$, $T$, and $S$. Let $j: \wedge^{2} A \rightarrow \wedge^{2} A /(S) \approx I \wedge I+I \wedge T+T \wedge T$ be the natural epimorphism. Then jod: $S \rightarrow I \wedge I+I \wedge T+T \wedge T$. However, in [2, pp. 577-8] Cartan shows that we may always assume $T$ chosen so that jod: $S \rightarrow I \wedge T=I \otimes T$, and so we are led to study linear transformations $\delta: S \rightarrow I \otimes T$. This was the viewpoint in my recent paper [5]. Observe that $\delta=j o d$ is linear over $R$. For, if $f \in R$, $\sigma \in S, d(f \sigma)=d f \wedge \sigma+f d \sigma$. Hence $\delta(f \sigma)=j o d(f \sigma)=j(d f \wedge \sigma)+$ $f j(d \sigma)=f j d(\sigma)=f \delta(\sigma)$, since $d f \wedge \sigma \in(S)$.

Received November 10, 1964. 
Historically the use of $R$ instead of the more customary ring of $C^{\infty}$-functions was because the supporting partial differential equations theory exists only in the analytic case. But $R$ also has the property of being an integral domain. If $F$ denotes its quotient field, then we may consider $S, I, T, I \otimes T$ as vector spaces over $F$, and $\delta$ as a linear transformation on these vector spaces.

This will enable us to use the techniques of linear algebra to define Cartan's numerical invariants and his concept of "involutive". These are usually defined pointwise and then their maximum values used in studying the global systems. Cartan wrote of "generic" properties [3], and we return to his concept, defining a generic system by extending the field $F$ in a suitable manner. In $\S 4$ these methods are applied to the study of systems which reproduce themselves under prolongations. In $\S 5$ the higher order prolongations of a system are identified as certain mappings on tensor products of the original spaces. These results are applied in $\S 6$ to given an elementary proof of Kuranishi's prolongation theorem. In this paper it seemed more convenient to study the dual of $\delta$, defined below.

\section{Differential systems and prolongations.}

Definition 1.1. A (differential) system $(I, T, d, S$ ) consists in 3 vector spaces $I, T, S$ over a field $F$ and a linear transformation $d: I \otimes T \rightarrow S$. The space $I$ is the vector space of independent variables.

Denote by $i$ the identity transformation on various spaces and by $j: I \wedge I \rightarrow I \otimes I$ the dual of the canonical epimorphism $I^{*} \otimes I^{*} \rightarrow I^{*} \wedge I^{*}$.

Definition 1.2. $(I, U, \delta, T)$ is a prolongation of $(I, T, d, S)$ if $d(i \otimes \delta)(j \otimes i)(I \wedge I \otimes U)=0$. That is, the composition

$$
(I \wedge I) \otimes U \stackrel{j \otimes i}{\longrightarrow} I \otimes(I \otimes U) \stackrel{i \otimes \delta}{\longrightarrow} I \otimes T \stackrel{d}{\longrightarrow} S
$$

is zero.

DeFinition 1.3. The (total, normal) prolongation is defined as follows. Let

$$
V=\{\phi \in \operatorname{Hom}(I, T) \mid d(i \otimes \phi) j(I \wedge I)=0\} .
$$

There is a natural linear transformation $\delta: I \otimes V \rightarrow T$ satisfying, for $\omega \in I, \phi \in V, \delta(\omega \otimes \phi)=\phi(\omega)$.

In [5] the author showed that the normal prolongation is a prolongation and in fact maximal in a certain sense among all prolongations. In the present paper all prolongations will be normal, so we shall omit 
the qualification "normal".

2. Involution. E. Cartan characterized algebraically exterior differential systems of equations which could be solved by the CauchyKowalewski system [4]. Systems satisfying these algebraic conditions are called "involutive". We shall define this notion and prove some results about it.

Let $(I, T, d, S)$ be a system. Let $\omega_{1}, \cdots, \omega_{p}$ be a basis of $I$. Let $I_{h}$ denote the subspace of $I$ spanned by $\omega_{1}, \cdots, \omega_{l}$.

DeFinition 2.1. An integral element of dimension $h$ (relative to $\left.\omega_{1}, \cdots, \omega_{p}\right)$ is a linear transformation $\phi_{h}: I_{h} \rightarrow T$ such that $d\left(i \otimes \phi_{h}\right) j\left(I_{h} \wedge I_{h}\right)=0$. This means that for all $i \leqq h, j \leqq h$,

$$
d\left(\omega_{i} \otimes \dot{\phi}_{h}\left(\omega_{j}\right)\right)=d\left(\omega_{j} \otimes \phi_{h}\left(\omega_{i}\right)\right) .
$$

Let $\mathscr{I}_{h}$ denote the linear space of integral elements of dimension $h$. Then $\mathscr{F}_{1}$ and $\mathscr{F}_{p}$ are isomorphic to $T$ and $V$, respectively (see Definition 1.3). There are natural linear transformations $\rho_{h+1}: \mathscr{I}_{h+1} \rightarrow \mathscr{I}_{h}$ obtained by restricting elements in $\mathscr{J}_{h+1}$ to $I_{h}, h=1,2, \cdots, p-1$.

Definition 2.2. $(I, T, d, S)$ is $h$-involutive (relative to $\omega_{1}, \cdots, \omega_{p}$ ) if $\rho_{h+1}$ is a epimorphism. It is involutive (relative to $\omega_{1}, \cdots, \omega_{p}$ ) if it is $h$-involutive for each $h=1,2, \cdots, p-1 . \quad(I, T, d, S)$ is involutive if it is involutive relative to at least one basis $\omega_{1}, \cdots, \omega_{p}$.

Let $\mathscr{P}_{h}$ be the kernel of $\rho_{h-1}$, let $\tau_{h}\left(\omega_{1}, \cdots, \omega_{p}\right)=\tau_{h}(\omega)=\operatorname{dim} \mathscr{P}_{h}$, $\tau_{0}(\omega)=\operatorname{dim} T$. Then $\operatorname{dim} \mathscr{F}_{h-1} \leqq \tau_{h}(\omega)+\operatorname{dim} \mathscr{I}_{h}$, and equality holds if and only if $(I, T, d, S)$ is $h$-involutive (relative to $\left.\omega_{1}, \cdots, \omega_{p}\right)$. Hence one can prove the following:

\section{THEOREM 2.1.}

$$
\operatorname{dim} V \leqq \tau_{0}(\omega)+\tau_{1}(\omega)+\cdots+\tau_{p_{-1}}(\omega),
$$

and equality holds if and only if $(I, T, d, S)$ is involutive (relative to $\left.\omega_{1}, \cdots, \omega_{p}\right)$.

Now, let $\omega_{1}, \cdots, \omega_{p}$ be any fixed basis. Let $F(x)$ be the field of rational functions over $F$ in variables $x_{j}^{i} ; i, j=1,2, \cdots, p$. Let $I^{*}=$ $I \otimes_{F} F(x), T^{*}=T \otimes_{F} F(x), S^{*}=S \otimes_{F} F(x)$, regarded as vector spaces over $F(x)$, and extend $d$ to be a linear transformation over $F(x)$ on $I^{*} \otimes_{F^{(x)}} T^{*}$ into $S^{*}$. Let

$$
\mu_{k}=\sum_{i=1}^{p} x_{k}^{i} \omega_{i}, \quad k=1, \cdots, p .
$$


Then $\mu_{1}, \cdots, \mu_{p}$ form a basis of $I^{*}$. We intend to use $\mu_{1}, \cdots, \mu_{p}$ as a "generic" basis of $I$. Let $I_{h}^{*}$ be the subspace spanned by $\omega_{1}, \cdots$, $\omega_{h}$, and define $\mathscr{F}_{h}^{*}, \rho_{h+1}^{*}, \mathscr{P}_{h}^{*}, \tau_{h}^{*}$ as before.

Proposition 2.1. If $\tau_{h}(\bar{\omega})$ is defined relative to some basis $\bar{\omega}_{1}, \cdots$, $\bar{\omega}_{p}$ then $\tau_{h}(\bar{\omega}) \leqq \tau_{h}^{*}$, and equality holds for some basis $\bar{\omega}_{1}, \cdots, \bar{\omega}_{p}$.

Proof. Let $\pi_{1}, \cdots, \pi_{q}$ and $\theta_{1}, \cdots, \theta_{r}$ be bases of $T$ and $S$, respectively. Then $d\left(\omega_{i} \otimes \pi_{\lambda}\right)=a_{i \lambda}^{u} \theta_{u}$. If $\phi \in \mathscr{P}_{h}$, then $\phi\left(\mu_{i}\right)=0$ for $i \leqq h$, and $\phi\left(\mu_{h+1}\right)=\sum_{\lambda=1}^{q} c^{\lambda} \pi_{\lambda}$, where

$$
\begin{aligned}
d(i \otimes \phi) j\left(\mu_{i} \wedge \mu_{k}\right) & =\sum_{\lambda=1}^{q} c^{\lambda} d\left(\mu_{i} \otimes \pi_{\lambda}\right) & \\
& =\sum_{k=1}^{p} \sum_{\lambda=1}^{q} c^{\lambda} x_{i}^{k} a_{k \lambda}^{u} \theta_{u}=0, & i \leqq h .
\end{aligned}
$$

Thus, the $c^{\lambda}$ must satisfy the $p r$ linear equations

$$
\sum_{k=1}^{p} \sum_{\lambda=1}^{q} c^{\lambda} x_{i}^{k} a_{k \lambda}^{u}=0, \quad \begin{aligned}
& i=1, \cdots, h \\
& u=1, \cdots, q .
\end{aligned}
$$

Conversely, every solution of these equations yields an elements of $\mathscr{P}_{h}^{*}$. $\tau_{h}^{*}$ is the number of linearly independent solutions.

Now suppose $\bar{x}_{j}^{i}$ are elements of $F$ such that $\bar{\omega}_{i}=\sum \bar{x}_{i}^{k} \omega_{k}$. Then the equations for members of $\mathscr{P}_{h}$ (relative to $\omega_{1}, \cdots, \omega_{p}$ ) are

$$
\sum_{k=1}^{p} \sum_{\lambda=1}^{q} c^{\lambda} \bar{x}_{i}^{k} \alpha_{k \lambda}^{u}=0, \quad i \leqq h .
$$

Hence $\tau_{h}(\bar{\omega}) \leqq \tau_{h}^{*}$.

We also see from this that it is possible to choose $\bar{x}_{i}^{k} \in F$ so that $\tau_{h}(\bar{\omega})=\tau_{h}^{*}$, all $h$.

Corollary 1. $\tau_{h}^{*}$ does not depend on $\omega_{1}, \cdots, \omega_{p}$.

We also observe from the above equations

CoROLlaRY 2. $\tau_{h}^{*} \leqq \tau_{h+1}^{*}$.

REMARK. This device of introducing $F(x)$ is often of practical value in computing $\tau_{h}$ in examples, since the results are independent. of $\omega_{1}, \cdots, \omega_{p}$.

Then if $\tau_{h}$ denotes the maximum of $\tau_{h}(\omega)$ over all bases $\omega_{1}, \cdots$, $\omega_{p}$ we have [3, p. 91].

THEOREM 2.1. $\operatorname{dim} V \leqq \tau_{0}+\cdots+\tau_{p_{-1}}$, and equality holds if and only if $(I, T, d, S)$ is involutive. 
To compare our definitions with those of Cartan, see [2, p. 579]. Matrix (14) there is the matrix associated with the equations for the $e^{\lambda}$ in Proposition 2.1. The $\sigma_{h}$ in Cartan's notation are related to our $\tau_{h}$ by $\tau_{h}=q-\left(\sigma_{1}+\cdots+\sigma_{k}\right)$.

3. Prolongations of involutive systems. We shall now give an elementary proof that the normal prolongation of an involutive system is also involutive [7]. Let $(I, T, d, S)$ be an involutive system relative to a basis $\omega_{1}, \cdots, \omega_{p}$ of $I$. The (normal) prolongation $(I, V$, $\delta, T)$ is defined by $V=\{\phi \in \operatorname{Hom}(I, T) \mid d(i \otimes \phi) j(I \wedge I)=0\}, \delta(\omega \otimes \phi)=$ $\phi(\omega)$. Let $I_{h}, \mathscr{J}_{h}, \mathscr{P}_{h}, \rho_{h+1}, \tau_{h}(\omega)$ be as before for the system $(I, T$, $d, S)$. The entities corresponding to these for $(I, V, \delta, T)$ will be denoted by $I_{h}, \mathscr{I}_{h}^{\prime}, \mathscr{P}_{k}^{\prime}, \rho_{h+1}^{\prime}, \tau_{h}^{\prime}(\omega)$, respectively.

Proposition 3.1. If $(I, T, d, S)$ is involutive (relative to $\omega_{1}, \cdots$, $\left.\omega_{p}\right)$, so is $(I, V, \delta, T)$.

Proof. We shall show that $\rho_{h-1}^{\prime}$ is an epimorphism for each $h=1, \cdots, p-1$. Let $\tilde{\phi} \in \mathscr{I}_{h}{ }^{\prime}$. We wish to define $\tilde{\phi}$ on $\omega_{h+1}$ so that $\delta(i \otimes \tilde{\phi}) j\left(I_{h+1} \wedge I_{h+1}\right)=0$, given that $\delta(i \otimes \tilde{\phi}) j\left(I_{h} \wedge I_{h}\right)=0$. Thus, $\tilde{\phi}\left(\omega_{h+1}\right)$ must be chosen so that if $i \leqq h, \delta\left[\omega_{i} \otimes \tilde{\phi}\left(\omega_{h+1}\right)\right]=$ $\delta\left[\omega_{h+1} \otimes \tilde{\phi}\left(\omega_{i}\right)\right]$. By definition this requires that

$$
\tilde{\phi}\left(\omega_{h+1}\right)\left(\omega_{i}\right)=\tilde{\phi}\left(\omega_{i}\right)\left(\omega_{h+1}\right) \text {. }
$$

Hence, $\tilde{\phi}\left(\omega_{h+1}\right)$ is defined uniquely on $I_{h}$. In fact, if $i, k \leqq h$,

$$
\begin{aligned}
& d\left[i \otimes \tilde{\phi}\left(\omega_{h+1}\right) j\right]\left(\omega_{k} \wedge \omega_{i}\right) \\
&=d\left[\omega_{k} \otimes \tilde{\phi}\left(\omega_{h+1}\right)\left(\omega_{i}\right)\right]-d\left[\omega_{i} \otimes \tilde{\phi}\left(\omega_{h+1}\right)\left(\omega_{k}\right)\right] \\
&= d\left[\omega_{k} \otimes \tilde{\phi}\left(\omega_{i}\right)\left(\omega_{h+1}\right)\right]-d\left[\omega_{i} \otimes \tilde{\phi}\left(\omega_{k}\right)\left(\omega_{h+1}\right)\right] \text { by }(1) \\
&= d\left[\omega_{k} \otimes \tilde{\phi}\left(\omega_{i}\right)\left(\omega_{h+1}\right)\right]-d\left[\omega_{h+1} \otimes \tilde{\phi}\left(\omega_{i}\right)\left(\omega_{k}\right)\right] \\
&+d\left[\omega_{h+1} \otimes \tilde{\phi}\left(\omega_{i}\right)\left(\omega_{k}\right)\right]-d\left[\omega_{h+1} \otimes \tilde{\phi}\left(\omega_{k}\right)\left(\omega_{i}\right)\right] \\
&+d\left[\omega_{h+1} \otimes \tilde{\phi}\left(\omega_{k}\right)\left(\omega_{i}\right)\right]-d\left[\omega_{i} \otimes \tilde{\phi}\left(\omega_{k}\right)\left(\omega_{h+1}\right)\right] \\
&= d\left[i \otimes \tilde{\phi}\left(\omega_{i}\right)\right] j\left(\omega_{k} \wedge \omega_{h+1}\right) \\
&+d\left\{\omega_{h-1} \otimes \delta(i \otimes \tilde{\phi}) j\left(\omega_{i} \wedge \omega_{k}\right)\right\}-d\left[i \otimes \tilde{\phi}\left(\omega_{k}\right)\right] j\left(\omega_{i} \wedge \omega_{h+1}\right) \\
&= 0,
\end{aligned}
$$

since $\tilde{\phi}\left(\omega_{i}\right)$ and $\tilde{\phi}\left(\omega_{k}\right)$ belong to $V$ and $\delta(i \otimes \tilde{\phi}) j\left(I_{h} \wedge I_{h}\right)=0$. Thus $\tilde{\phi}\left(\omega_{h+1}\right) \in \mathscr{I}_{h}$. Since $(I, T, d, S)$ is involutive, $\rho_{h+i}$ are epimorphisms, so there exists an extension of $\tilde{\phi}\left(\omega_{h+1}\right)$ to all of $I$, this extension being in $V$. In this way $\tilde{\phi}$ is defined on $I_{h+1}$ into $V$ and becomes an element of $\mathscr{I}_{h+1}^{\prime}$. Thus $\rho_{h+1}^{\prime}$ is an epimorphism for all $h$.

Proposition 3.2. If $(I, T, d, S)$ is involutive, $\mathscr{P}_{h}^{\prime}$ is isomorphic 
to the direct sum

$$
\mathscr{P}_{h}+\mathscr{P}_{h+1}+\cdots+\mathscr{P}_{p-1} .
$$

Hence $\tau_{h}^{\prime}(\omega)=\sum_{j=h}^{p-1} \tau_{j}(\omega)$.

Proof. Let $\tilde{\phi} \in \mathscr{P}_{h}^{\prime}$. Then $\tilde{\phi}: I_{h+1} \rightarrow V, \tilde{\phi}\left(I_{h}\right)=0$, and $\delta[i \otimes \phi] j\left(I_{h+1} \wedge\right.$ $\left.I_{h+1}\right)=0$. If $i \leqq h, \delta[i \otimes \tilde{\phi}] j\left(\omega_{i} \wedge \omega_{h+1}\right)=\delta\left[\omega_{i} \otimes \tilde{\phi}\left(\omega_{h+1}\right)\right]=\widetilde{\phi}\left(\omega_{h+1}\right)\left(\omega_{i}\right)$ $=0$. Thus, $\tilde{\phi}\left(\omega_{h+1}\right)$ is in $V$ and is zero on $I_{h}$. Let $\mathscr{Q}_{h}=\left\{\phi \in V \mid \phi\left(I_{h}\right)=0\right\}$. Then $\tilde{\phi}\left(\omega_{h+1}\right) \in \mathscr{Q}_{h}$, and conversely every element of $\mathscr{Q}_{h}$ corresponds to a unique member of $\mathscr{P}_{h}^{\prime}$. Hence $\mathscr{P}_{h}^{\prime}$ is isomorphic to $\mathscr{Q}_{h}$.

We have an exact sequence $0 \rightarrow \mathscr{Q}_{h+1} \rightarrow \mathscr{Q}_{h} \rightarrow \mathscr{P}_{h}$, and when $(I, T$, $d, S)$ is involutive the last linear transformation is an epimorphism. Hence

$$
\mathscr{Q}_{h} \approx \mathscr{P}_{h}+\mathscr{Q}_{h+1} \approx \cdots \approx \mathscr{P}_{h}+\mathscr{P}_{h+1}+\cdots+\mathscr{P}_{p-1} .
$$

Observe that even when $(I, T, d, S)$ is not involutive one has an exact sequence $0 \rightarrow \mathbb{Q}_{h+1} \rightarrow \mathbb{Q}_{h} \rightarrow \mathscr{P}_{h}$, and $\mathbb{Q}_{h} \approx \mathscr{P}_{h}^{\prime}$.

THEOREM 3.1. If $\tau_{h}^{\prime}(\omega), h=0,1, \cdots, p-1$, are the numbers associated with the normal prolongation $(I, V, \delta, T)$ of $(I, T, d, S)$, then

$$
\tau_{h}^{\prime}(\omega) \leqq \tau_{h}(\omega)+\tau_{h+1}(\omega)+\cdots+\tau_{p-1}(\omega) .
$$

If $(I, T, d, S)$ is involutive, then so is $(I, V, \delta, T)$, and equality holds in the above relation.

4. Self-reproducing systems. The methods will now be applied to study certain systems which remain unchanged under prolongations. Let $(I, T, d, S)$ be a given system, $(I, V, \delta, T)$ its prolongation, and $\omega_{1}$ a nonzero element of $I$. Define $f: V \rightarrow T$ be $f(\phi)=\phi\left(\omega_{1}\right)$. Let $F=$ $i \otimes f: I \otimes V \rightarrow I \otimes T$. Define $G: T \rightarrow S$ by $G(\pi)=d\left(\omega_{1} \otimes \pi\right)$.

$$
\text { Proposition 4.1. } \quad G \delta=d F \text {. }
$$

Proof. We have $G \delta(\omega \otimes \phi)=d\left(\omega_{1} \otimes \phi(\omega)\right)$ and $d F(\omega \otimes \phi)=$ $d\left(\omega \otimes \phi\left(\omega_{1}\right)\right) . \quad$ Since $\quad \phi \in V, \quad 0=d(i \otimes \phi) j\left(\omega \wedge \omega_{1}\right)=d\left(\omega \otimes \phi\left(\omega_{1}\right)\right)=$ $d\left(\omega_{1} \otimes \phi(\omega)\right)$.

Proposition 4.2. If $(I, T, d, S)$ is involutive (relative to $\omega_{1}, \cdots$, $\left.\omega_{p}\right)$, then $F$ is an epimorphism.

Proof. Given $\omega \otimes \pi \in I \otimes T$, let $\phi\left(\omega_{1}\right)=\pi, \phi \in \mathscr{I}_{1}$. Then $\phi$ may be extended to $I_{2}$, hence to $I_{3}$, etc., hence to $I$. Thus, there is an element $\phi \in V$ such that $\phi\left(\omega_{1}\right)=\pi$. Then $F(\omega \otimes \phi)=\omega \otimes \pi$. 
Proposition 4.3. If $(I, T, d, S)$ is involutive (relative to $\omega_{1}, \cdots$, $\left.\omega_{p}\right)$ and $\tau_{1}=0$, then $F$ is an endomorphism.

Proof. The exactness of $0 \rightarrow \mathscr{P}_{h} \rightarrow \mathscr{I}_{h+1} \rightarrow \mathscr{I}_{h} \rightarrow 0$ implies that the restriction linear transformation $\rho_{h+1}$ is an isomorphism for all $h \geqq 1$, since Corollary 2 to Proposition 2.1 implies $\mathscr{P}_{h}=0$ for all $h \geqq 1$. Then given $\omega_{1}$ and $\pi$, there exists a unique $\phi \in V$ such that $\phi\left(\omega_{1}\right)=\pi$.

Define $g: T \rightarrow V$ by $g(\pi)=\phi$ provided $\phi\left(\omega_{1}\right)=\pi$ is an isomorphism by the above remarks. Thus, $\operatorname{dim} I \otimes V=\operatorname{dim} I \otimes T=\operatorname{dim} I \otimes V-$ $\operatorname{dim}(\operatorname{ker} F)$. Hence $\operatorname{ker} F=0$.

Proposition 4.4. If $(I, T, d, S)$ is involutive (relative to $\omega_{1}, \cdots$, $\left.\omega_{p}\right)$ and $d$ is an epimorphism, then $G$ is an epimorphism.

Proof. $\delta$ is an epimorphism; for, given $\pi \in T$, then as in Proposition 4.2 we can choose $\phi \in V$ so that $\phi\left(\omega_{1}\right)=\pi$. Hence $\delta\left(\omega_{1} \otimes \phi\right)=\pi$. $F$ is an epimorphism by Proposition 4.3, so by Proposition 4.1. $G$ must be an epimorphism.

Proposition 4.5. If $(I, T, d, S)$ is involutive (relative to $\omega_{1}, \cdots$, $\left.\omega_{p}\right), d$ is an epimorphism, and $\operatorname{dim} T=\operatorname{dim} S$, then $G$ is an endomorphism.

Proof. Immediate consequence of Proposition 4.4.

Definition 4.1. Two systems $(I, T, d, S)$ and $\left(I^{\prime}, T^{\prime}, d^{\prime}, S^{\prime}\right)$ are equivalent if there exist isomorphisms $f: T \rightarrow T^{\prime}$ and $G: S \rightarrow S^{\prime}$ such that if $F=i \otimes f: I \otimes T \rightarrow I \otimes T^{\prime}$, then $G d=d^{\prime} F$.

We have proved the following

THEOREM 4.1. If $(I, T, d, S)$ is involutive (relative to $\left.\omega_{1}, \cdots, \omega_{p}\right)$, $\tau_{1}=0, d$ is an epimorphism, and $\operatorname{dim} T=\operatorname{dim} S$, then $(I, T, d, S)$ and $(I, V, \delta, T)$ are equivalent. Thus, under these conditions the prolongation reproduces a system equivalent to the original system.

5. Higher order prolongations. In this section we study the process of iterated prolongations and identify the system obtained by prolonging $(I, T, d, S)$ a number of times. This will be used in $\S 6$ to prove a theorem of Cartan and Kuranishi that every system becomes involutive after a sufficient number of prolongations [6].

If $\sigma$ is any permutation of $1,2, \cdots, k$, we denote by $p_{\sigma}$ the automorphism on $\otimes^{k} I$ which satisfies $p_{\sigma}\left(\omega_{1} \otimes \cdots \otimes \omega_{k}\right)=\omega_{\sigma(1)} \otimes \cdots \otimes \omega_{\sigma(k)}$. A linear transformation $\eta: \otimes{ }^{k} I \rightarrow T$ is called symmetric when $\eta=\eta p_{\sigma}$ for every $\sigma$. 
DEFINITION 5.1. Let

$$
\begin{aligned}
V^{k}= & \left\{\eta \in \operatorname{Hom}\left(\otimes{ }^{k} I, T\right) \mid \eta\right. \text { is symmetric, and } \\
& \left.d(i \otimes \eta)(j \otimes i)\left[(I \wedge I) \otimes{ }^{k-1} I\right]=0\right\}
\end{aligned}
$$

if $k>0$, and $V^{0}=T$. If $k>1, \delta^{k}: I \otimes V^{k} \rightarrow V^{k-1}$ is defined such that if $\omega \in I, \eta \in V^{k}, \gamma \in \otimes^{k-1} I$, then $\left[\delta^{k}(\omega \otimes \eta)\right](\gamma)=\eta(\gamma \otimes \omega)$. Also, $\delta^{1}=\delta$ of Definition 1.3. The system $\left(I, V^{k}, \delta^{k}, V^{k-1}\right)$ is called the kth (total, normal) prolongation of $(I, T, d, S)$.

It is a computation to check that $\delta^{k}$ maps into $V^{k-1}$. The second condition in the definition of $V^{k}$ means that if $\omega_{0}, \omega_{1}, \cdots, \omega_{k}$ are in $I$, then

$$
d\left[\omega_{0} \otimes \eta\left(\omega_{1} \otimes \omega_{2} \otimes \cdots \otimes \omega_{k}\right)\right]=d\left[\omega_{1} \otimes \eta\left(\omega_{0} \otimes \omega_{2} \otimes \cdots \otimes \omega_{k}\right)\right] .
$$

Theorem 5.1. (I, $\left.V^{k}, \delta^{k}, V^{k-1}\right)$ is equivalent to the system obtained by successively prolonging $(I, T, d, S) k$ times.

Proof. For induction, one shows $\left(I, V^{k+1}, \delta^{k+1}, V^{k}\right)$ is equivalent to the prolongation of $\left(I, V^{k}, \delta^{k}, V^{k-1}\right)$. Let

$$
U=\left\{\phi \in \operatorname{Hom}\left(I, V^{k}\right) \mid \delta^{k}(i \otimes \phi) j(I \wedge I)=0\right\},
$$

let $\mu: I \otimes U \rightarrow V^{k}$ be defined by $\mu(\omega \otimes \phi)=\phi(\omega)$. Then it must be shown that $\left(I, U, \mu, V^{k}\right)$ is equivalent to $\left(I, V^{k+1}, \delta^{k+1}, V^{k}\right)$. In fact, one can define $f: U \rightarrow V^{k+1}$ as follows: if $\omega_{1}, \cdots, \omega_{k+1} \in I, \phi \in U$, let $f(\phi)\left(\omega_{1} \otimes \cdots \otimes \omega_{k+1}\right)=\phi\left(\omega_{1}\right)\left(\omega_{2} \otimes \cdots \otimes \omega_{k+1}\right)$. Then a computation shows that $f$ is well-defined. Also $f$ has an inverse $g: V^{k+1} \rightarrow U$ constructed similarly. Moreover, $\delta^{k+1}(\omega \otimes f(\phi))(\gamma)=f(\phi)(\omega \otimes \gamma)=\phi(\omega)(\gamma)=$ $\mu(\omega \otimes \phi)$. Hence letting $F=i \otimes f: I \otimes U \rightarrow I \otimes V^{k+1}$ and $G=$ identity: $V^{k} \rightarrow V^{k}$, we have $\delta^{k+1} F=G \mu$.

Next we shall identify integral elements of the system $\left(I, V^{k}, \delta^{k}\right.$, $\left.V^{k-1}\right)$. Let $\omega_{1}, \cdots, \omega_{p}$ be a basis of $I$. Let $I_{h}$ be spanned by $\omega_{1}, \cdots, \omega_{h}$.

Definition 5.2. Let, for $1 \leqq k \leqq p$,

$$
\begin{aligned}
\mathscr{I}_{h}{ }^{k}= & \left\{\phi \in \operatorname{Hom}\left(\otimes{ }^{k} I \otimes I_{h}, T\right) \mid \phi\right. \text { is symmetric and } \\
& \left.d(i \otimes \phi)(j \otimes i)\left[(I \wedge I) \otimes{ }^{k-1} I \otimes I_{h}\right]=0\right\} .
\end{aligned}
$$

("Symmetric" refers to all possible symmetries preserving $\otimes{ }^{k} I \otimes I_{h}$ ). Let $\rho_{h+1}^{k}: \mathscr{J}_{h+1}^{k} \rightarrow \mathscr{F}_{h}^{k}$ be the restriction linear transformation, with kernel $\mathscr{P}_{h}^{k}$. Let

$$
\begin{aligned}
& \mathbb{Q}_{h}^{k}=\left\{\eta \in V^{k+1} \mid \eta\left(\otimes{ }^{k} I \otimes I_{h}\right)=0\right\} \text { if } h>0, \\
& \mathbb{Q}_{0}^{p k}=V^{k+1} .
\end{aligned}
$$


Observe that $\mathscr{F}_{0}^{k}=V^{k+1}, \mathscr{Q}_{p}^{k}=0$.

THEOREM 5.2. $\mathscr{I}_{h}^{k}$ is isomorphic to the space of integral elements of dimension $h$ (relative to $\left.\omega_{1}, \cdots, \omega_{p}\right)$ on $\left(I, V^{k}, \delta^{k}, V^{k-1}\right)$. If we identify these isomorphic spaces, $\rho_{h+1}^{k}$ corresponds to the restriction linear transformation, and $\mathscr{P}_{h}^{k}$ and $\mathscr{Q}_{h}^{k-1}$ are isomorphic.

Proof. The first two assertions follow by a computation. The last isomorphism is defined as follows: $\phi \in \mathscr{P}_{h}^{k}$ is identified with the element of $\mathscr{Q}_{h}^{k-1}$ whose value on $\gamma \in \otimes{ }^{k} I$ is $\phi\left(\gamma \otimes \omega_{h+1}\right)$.

Proposition 5.1. For any element $\omega_{0} \in I$, there exists a linear transformation $\lambda^{k}\left(\omega_{0}\right): \mathbb{Q}_{h}^{k} \rightarrow \mathbb{Q}_{h}^{k-1}$ which satisfies, for $\eta \in \mathbb{Q}_{h}^{k}, \gamma \in \bigotimes^{k} I$,

$$
\left[\lambda^{k}\left(\omega_{0}\right)(\eta)\right](\gamma)=\eta\left(\gamma \otimes \omega_{0}\right) .
$$

If $\omega_{0}=\omega_{h+1}$, the kernel of $\lambda^{k}\left(\omega_{0}\right)$ is $\mathbb{Q}_{h+1}^{k}$.

Proof. A computation shows that $\lambda^{k}\left(\omega_{0}\right)$ is well-defined. The second assertion follows from the definitions.

THEOREM 5.3. $\left(I, V^{k}, \delta^{k}, V^{k-1}\right)$ is involutive if and only if $V^{k+1}$ is isomorphic to the direct sum

$$
\mathbb{Q}_{0}^{k-1}+\mathbb{Q}_{1}^{k-1}+\cdots+\mathbb{Q}_{p-1}^{k-1} \approx V^{k-1},
$$

or, if and only if $\lambda^{k}\left(\omega_{h+1}\right)$ is an epimorphism for $h=0,1, \cdots, p-1$.

Proof. The first condition follows from Theorem 2.1 and the last statement of Theorem 5.2. Next, $\lambda^{k}\left(\omega_{k+1}\right)$ is an epimorphism if and only if

$$
\mathbb{Q}_{h}^{k} \approx \mathbb{Q}_{h}^{k-1}+\mathbb{Q}_{h+1}^{k}
$$

and $\mathscr{Q}_{0}^{k}=V^{k+1}$. This implies isomorphism (2). If some $\lambda^{k}\left(\omega_{h+1}\right)$ were not an epimorphism, the dimensions of the various spaces would preclude any such isomorphism (2).

6. The prolongation theorem. We shall next prove that if $(I, T, d, S)$ is any given system, there exists an integer $k_{0}$ such that the $k^{\text {th }}$ prolongation $\left(I, V^{k}, \delta^{k}, V^{k-1}\right)$ is involutive for all $k \geqq k_{0}$. In fact, we shall show the existence of one basis $\omega_{1}, \cdots, \omega_{p}$, relative to which all $\left(I, V^{k}, \delta^{k}, V^{k-1}\right)$ are involutive for $k \geqq$ some $k_{0}$.

To show this it is sufficient to show that $\lambda^{k}\left(\omega_{h+1}\right): \mathbb{Q}_{h}^{k} \rightarrow \mathbb{Q}_{h}^{k-1}$ is an epimorphism for all $k \geqq k_{0}$, by Theorem 5.3. This will be done inductively, choosing $\omega_{h+1}$ after $\omega_{1}, \cdots, \omega_{h}$ such that $\lambda^{k}\left(\omega_{h+1}\right)$ is an 
epimorphism. Choice of $\omega_{h+1}$ is accomplished by relating this problem to one in classical ideal theory. Let us temporarily extend $\omega_{1}, \cdots, \omega_{k}$ to any basis $\omega_{1}, \cdots, \omega_{p}$ of $I$. Then $\bar{\omega}_{h+1}$ will be chosen later so that using $\bar{\omega}_{h+1}, \lambda^{k}\left(\bar{\omega}_{h+1}\right)$ will be an epimorphism.

Let $F\left[x_{1}, \cdots, x_{p}\right]^{(k)}$ be the vector space of polynomials in $x_{1}, \cdots, x_{p}$ over $F$ which are homogenous of degree $k$. Define an epimorphism $\nu: \otimes{ }^{k} I \rightarrow F\left[x_{1}, \cdots, x_{p}\right]^{(k)}$ so that $\nu\left(\omega_{i_{1}} \otimes \cdots \otimes \omega_{i_{k}}\right)=x_{i_{1}} \cdots x_{i_{k}}$. If $\eta \in V^{k}, \eta$ is symmetric, hence $\eta($ ker $\nu)=0$. It follows that $\eta$ induces a unique linear transformation $\bar{\eta}$ on $F\left[x_{1}, \cdots, x_{p}\right]^{(k)}$ into $T$.

These $\bar{\eta}$ can be considered linear transformations on $F\left[x_{1}, \cdots\right.$, $\left.x_{p}\right]^{(k)} \otimes T^{*}$ into $F$, where $T^{*}$ is the dual of $T$. If $\operatorname{dim} T=q$ and $\pi_{1}^{*}, \cdots, \pi_{q}^{*}$ is a basis of $T^{*}$, we can identity $F\left[x_{1}, \cdots, x_{p}\right]^{(k)} \otimes T^{*}$ with the vector space $F\left[x_{1}, \cdots, x_{p}, t_{1}, \cdots, t_{q}\right]^{(k, 1)}=F[x, t]^{(k, 1)}$ of polynomials in $x_{1}, \cdots, x_{p}, t_{1}, \cdots, t_{q}$ which are homogenous of degree $k$ in the $x_{i}$ and degree 1 in the $t_{\lambda}$. Then $V^{k}$ is identified with a subspace of the dual space of $F[x, t]^{(k, 1)}$. Let

$$
\left.A\left(V^{k}\right)=\left\{X \in F[x, t]^{(k, 1}\right) \mid \phi(X)=0 \text { for every } \phi \in V^{k}\right\} .
$$

Next, consider $\mathbb{Q}_{h}^{k-1}$, consisting of all members of $V^{k}$ which are zero on $\otimes{ }^{k} I \otimes I_{h}$. If $\left(x_{1}, \cdots, x_{h}\right)$ is the ideal in $F[x, t]$ generated by $x_{1}, \cdots, x_{h}$; and if $\left(x_{1}, \cdots, x_{h}\right)^{(k, 1)}$ is its subspace of elements homogenous of degree $k$ in $x$ and 1 in $t$, let $a\left[\left(x_{1}, \cdots, x_{h}\right)^{(k, 1)}\right]=\left\{\phi \in \operatorname{Hom}\left(F[x, t]^{(k, 1)}, F\right) \mid\right.$ $\left.\phi\left[\left(x_{1}, \cdots, x_{h}\right)^{(k, 1)}\right]=0\right\}$ (the dual space to $\left.\left(x_{1}, \cdots, x_{h}\right)^{(k, 1)}\right)$.

Then by definition

$$
\mathbb{Q}_{h}^{k-1}=V^{k} \cap a\left[\left(x_{1}, \cdots, x_{h}\right)^{(k, 1)}\right] .
$$

It follows that

$$
A\left(\mathbb{Q}_{h}^{k-1}\right)=A\left(V^{k}\right)+\left(x_{1}, \cdots, x_{h}\right)^{(k, 1)} .
$$

Proposition 6.1. $\quad x_{i} A\left(\mathbb{Q}_{h}^{k-1}\right) \subset A\left(\mathscr{Q}_{h}^{k}\right)$.

Let $\bar{\omega}$ denote the element of $F[x, t]^{(1,0)}$ corresponding to $\omega \in I$. Then $\lambda^{k}(\omega)$ is an epimorphism if and only if the following holds: whenever $\bar{\omega} \gamma \in A\left(\mathbb{Q}_{h}^{k}\right)$, then $\gamma \in A\left(\mathscr{Q}_{h}^{k-1}\right)$.

Proof. $\lambda^{k}(\omega)$ is the dual of the linear transformation $r_{\bar{\omega}}: F[x, t]^{(k, 1)} \rightarrow$ $F[x, t]^{(k+1,1)}$ defined by $r_{\bar{\omega}}(\gamma)=\gamma \omega$. Since $\lambda^{k}(\omega)$ carries $\mathbb{Q}_{h}^{k}$ into $\mathbb{Q}_{h}^{k-1}$, $r_{\bar{x}_{i}}\left(A\left(\mathbb{Q}_{h}^{k-1}\right)\right) \subset A\left(\mathbb{Q}_{h}^{k}\right)$. Hence the first assertion. The second remark also follows from vector space theory.

Now let $A_{h}$ be the ideal in $F[x, t]$ generated by $A\left(\mathscr{Q}_{h}^{k}\right), k=1,2, \cdots$. By the last Proposition $A_{h}^{(k, 1)}=A\left(\mathscr{Q}_{h}^{k-1}\right)$. If $A_{0}$ is the ideal generated by $A\left(V^{k}\right), k=1,2, \cdots$, we have $A_{h}=A_{0}+\left(x_{1}, \cdots, x_{h}\right)$. 
Proposition 6.2. There exists an element $\omega \in F[x, t]^{(1,0)}$ and an integer $k_{0}$ such that if $k \geqq k_{0}$, then $\bar{\omega} \gamma-A\left(\mathbb{Q}_{h}^{k}\right)$ implies $\gamma \in A\left(\mathbb{Q}_{h}^{k-1}\right)$.

Proof. Since $A_{h}$ is an ideal in a Noetherian ring it has a decomposition $A_{h}=q_{1} \cap \cdots \cap q_{t}$ into primary ideals with distinct belonging prime ideals $p_{1}, \cdots, p_{t}$ such that

$$
\left(p_{i}\right)^{\sigma_{i}} \subset q_{i},
$$$$
i=1, \cdots, t \text {. }
$$

Let $k_{0}>\max \left(\sigma_{1}, \cdots, \sigma_{t}\right)$.

Now suppose $p_{i}^{(1,0)}=F[x, t]^{(1,0)}$. Then $\left(p_{i}^{k}\right)^{(k, 1)}=F[x, t]^{(k, 1)}$. Hence if $k \geqq k_{0}>\sigma_{i}, F[x, t]^{(k, 1)}=\left(q_{i}\right)^{(k, 1)}$, so $\bar{\omega} \gamma \in q_{i}^{(k, 1)}$ implies $\gamma \in q_{i}^{(k-1,1)}$.

Now suppose $p_{1}^{(1,0)}, \cdots, p_{s}^{(1,0)}$ contain all $F[x, t]^{(1,0)}$, while $p_{s+1}^{(10)}, \cdots$, $p_{t}^{(1,0)}$ do not. Each $p_{s+\jmath}^{(10)}$ is a proper subspace of $F[x, t]^{(1,0)}$, so we can choose $\bar{\omega} \in F[x, t]^{(1,0)}$ not in any $p_{s+j}^{(1,0)}, j=1, \cdots, t-s$. Then if $\bar{\omega} \gamma \in q_{s+i}^{(k, 1)}, \bar{\omega} \notin p_{s+j}$, hence $\gamma \in q_{s+i}^{(k-1,1)}$.

For this $\bar{\omega}$, if $\bar{\omega} \gamma \in A\left(\mathbb{Q}_{h}^{k}\right)=A_{h}^{(k+1,1)} \subset A_{h}=q_{1} \cap \cdots \cap q_{t}$, then $\gamma \in q_{1} \cap \cdots \cap q_{t}$, provided $k \geqq k_{0}$. Hence $\gamma \in A_{h}^{(k, 1)}=A\left(\mathbb{Q}_{h}^{k-1}\right)$.

COROLlaRY. There exists $\omega \in I$ and an integer $k_{0}$ such that $\omega$ is linearly independent of $\omega_{1}, \cdots, \omega_{h}$ and $\lambda^{k}(\omega)$ is an epimorphism for all $k \geqq k_{0}$.

Proof. If $F[x, t]^{(k, 1)} \neq A\left(\mathbb{Q}_{h}^{k-1}\right)$ for some $k \geqq k_{0}$, let $\gamma \in F[x, t]^{(k, 1)}-$ $A\left(\mathbb{Q}_{h}^{k-1}\right)$. If $\bar{\omega}$ chosen in Proposition 6.2 depends on $x_{1}, \cdots, x_{h}$, then $\bar{\omega} \gamma \in A\left(V^{k+1}\right)+\left(x_{1}, \cdots, x_{h}\right)^{(k+1,1)}=A\left(\mathbb{Q}_{h}^{k}\right)$, while $\gamma \notin A\left(\mathbb{Q}_{h}^{k-1}\right)$, a contradiction. Hence the $\omega \in I$ corresponding to $\bar{\omega}$ cannot depend on $\omega_{1}, \cdots, \omega_{h}$.

If $F[x, t]^{(k, 1)}=A\left(\mathbb{Q}_{h}^{k-1}\right)$ for all $k \geqq k_{0}$, any $\omega \in I$ will suffice.

Theorem 6.1. It is possible to choose a basis $\omega_{1}, \cdots, \omega_{h}$ of $I$ and an integer $k_{0}$ such that $\lambda^{k}\left(\omega_{h+1}\right)$ is an epimorphism for all $h$ and $k \geqq k_{0}$.

Proof. Having chosen $\omega_{1}, \cdots, \omega_{h}$, let $\omega_{h+1}$ be the $\omega$ of the previous Corollary.

THEOREM 6.2. (Kuranishi's Prolongation Theorem) For any system there exists an integer $k_{0}$ such that every $k$ th (normal) prolongation for $k \geqq k_{0}$ involutive.

\section{REFERENCES}

1. É. Cartan, Sur l'integration des systèmes d'équations aux différentielles totales, Ann. Éc. Normale 18 (1901), 241-311. Oeuvres II, 1. (Paris), 411-482. 
2. - Sur la structure des groups infinis de transformations, Ann. Ec. Normale 21 (1904), 153-206. Oeuvres II, 2. (Paris), 571-624.

3. - Les systèmes différentiels extèrieurs et leurs applications géométriques. (Paris), 1945.

4. R. Hermann, E. Cartan's theory of exterior differential systems, Notes of University of California, 1963.

5. H. H. Johnson, Algebraic aspects of prolongations. To appear in Proc. Amer. Math. Soc.

6. M. Kuranishi, On E. Cartan's prolongation theorem of exterior differential systems, Amer. J. Math. 79 (1957), 1-47.

7. Y. Matsushima, On a theorem concerning the prolongation of a differential system. Nagoya Math. J. 6 (1953), 1-16. 


\title{
PACIFIC JOURNAL OF MATHEMATICS
}

\author{
EDITORS
}

\section{H. SAMELSON}

Stanford University

Stanford, California

R. M. Blumenthal

University of Washington

Seattle, Washington 98105

\section{*J. DugundJI}

University of Southern California Los Angeles, California 90007

\section{RichaRd ARENS}

University of California

Los Angeles, California 90024

\section{ASSOCIATE EDITORS}
E. F. BECKENBACH
B. H. NEUManN
F. WOLF
K. YOSIDA

\section{SUPPORTING INSTITUTIONS}

\author{
UNIVERSITY OF BRITISH COLUMBIA \\ CALIFORNIA INSTITUTE OF TECHNOLOGY \\ UNIVERSITY OF CALIFORNIA \\ MONTANA STATE UNIVERSITY \\ UNIVERSITY OF NEVADA \\ NEW MEXICO STATE UNIVERSITY \\ OREGON STATE UNIVERSITY \\ UNIVERSITY OF OREGON \\ OSAKA UNIVERSITY \\ UNIVERSITY OF SOUTHERN CALIFORNIA
}

\author{
STANFORD UNIVERSITY \\ UNIVERSITY OF TOKYO \\ UNIVERSITY OF UTAH \\ WASHINGTON STATE UNIVERSITY \\ UNIVERSITY OF WASHINGTON \\ AMERICAN MATHEMATICAL SOCIETY \\ CHEVRON RESEARCH CORPORATION \\ TRW SYSTEMS \\ NAVAL ORDNANCE TEST STATION
}

\footnotetext{
Mathematical papers intended for publication in the Pacific Journal of Mathematics should be typewritten (double spaced). The first paragraph or two must be capable of being used separately as a synopsis of the entire paper. It should not contain references to the bibliography. Manuscripts may be sent to any one of the four editors. All other communications to the editors should be addressed to the managing editor, Richard Arens at the University of California, Los Angeles, California 90024 .

50 reprints per author of each article are furnished free of charge; additional copies may be obtained at cost in multiples of 50 .
}

The Pacific Journal of Mathematics is published monthly. Effective with Volume 16 the price per volume (3 numbers) is $\$ 8.00$; single issues, $\$ 3.00$. Special price for current issues to individual faculty members of supporting institutions and to individual members of the American Mathematical Society: $\$ 4.00$ per volume; single issues $\$ 1.50$. Back numbers are available.

Subscriptions, orders for back numbers, and changes of address should be sent to Pacific Journal of Mathematics, 103 Highland Boulevard, Berkeley 8, California.

Printed at Kokusai Bunken Insatsusha (International Academic Printing Co., Ltd.), No. 6, 2-chome, Fujimi-cho, Chiyoda-ku, Tokyo, Japan.

PUBLISHED BY PACIFIC JOURNAL OF MATHEMATICS, A NON-PROFIT CORPORATION

The Supporting Institutions listed above contribute to the cost of publication of this Journal, but they are not owners or publishers and have no responsibility for its content or policies.

* Paul A. White, Acting Editor until J. Dugundji returns. 


\section{Pacific Journal of Mathematics}

\section{Vol. 17, No. $3 \quad$ March, 1966}

Tsuyoshi Andô, Contractive projections in $L_{p}$ spaces ............... 391

Robert F. Brown, On a homotopy converse to the Lefschetz fixed point theorem ............................................... 407

Richard Albert Cleveland and Sandra Cleveland, On the multiplicative extension property................................ 413

Harold H. Johnson, An algebraic approach to exterior differential systems..................................... 423

Alan Cecil Lazer, The behavior of solutions of the differential equation

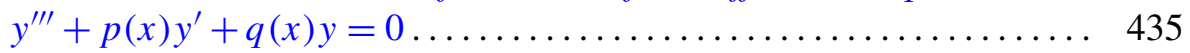

Judy Parr, Cohomology of cyclic groups of prime square order ......... 467

Donald Steven Passman, Groups whose irreducible representations have

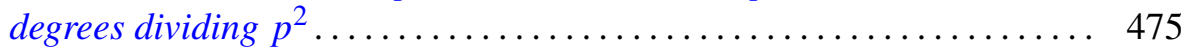

Ralph Tyrrell Rockafellar, Characterization of the subdifferentials of convex functions ........................................... 497

Donald Erik Sarason, Invariant subspaces and unstarred operator algebras...................................... 511

Donald Erik Sarason, Weak-star generators of $H^{\infty} \ldots \ldots \ldots \ldots \ldots \ldots . \ldots . \ldots$

Boris M. Schein, Homomorphisms and subdirect decompositions of semi-groups

Daniel Francis Shea, Jr., Functions analytic in a finite disk and having asymptotically prescribed characteristic

Zvi Ziegler, Generalized convexity cones 